\title{
Core intrinsic rotation behavior in ASDEX Upgrade Ohmic L-mode plasmas
}

\author{
R. M. McDermott, C. Angioni, G. D. Conway, R. Dux, \\ E. Fable, R. Fischer, T. Pütterich, F. Ryter, E. Viezzer \\ and the ASDEX Upgrade Team
}

Max Planck Institut für Plasmaphysik, Boltzmannstraße 2, D-85748 Garching, Germany

E-mail: Rachael.McDermott@ipp.mpg.de

\begin{abstract}
The ASDEX Upgrade intrinsic rotation database has been expanded to include a large number of measurements from Ohmic L-mode discharges covering a wide range of plasma densities, currents, and magnetic fields. This database was then used to study the rotation behavior across the transition from linear to saturated Ohmic confinement (LOC/SOC). At low collisionailty the plasma is in the LOC regime and the toroidal rotation profile is in the co-current direction. As the collisionality is increased and the plasma transitions from LOC to SOC, the core rotation decreases resulting in a hollow, counter-current profile. Even deeper in the SOC regime, however, a second reversal back toward the co-current direction occurs. Linear gyrokinetic calculations indicate that these reversals can not be explained by a transition from a trapped electron mode (TEM) to an ion temperature gradient (ITG) dominated regime. Rather, the analysis indicates that the intrinsic normalised rotation gradient, u', depends strongly on local plasma parameters, in particular on $R / L_{\text {ne }}$. Taken together with turbulent particle transport theory, these results suggest that the coto counter-current directed rotation reversal occurs in the TEM regime due to profile changes and not due to transition from TEM to ITG. The second reversal back to the co-current direction is explained by the reduction of $R / L_{n e}$ observed in ITG dominated plasmas. Lastly, a simple linear model for the residual stress assuming a fixed poloidal tilt angle of the turbulent eddies was applied to the database. This model is able to capture the main parameter dependencies observed in the data and demonstrates that at least two processes contributing to the poloidal tilt of the turbulent eddies are needed to reproduce the experimentally observed u' values: one proportional to the sign of the turbulence propagation and the second independent of $\omega_{r}$.
\end{abstract}

\section{Introduction}

Intrinsic rotation in tokamak plasmas, with its complex behavior and diverse phenomenology, has long presented the fusion community with an intriguing puzzle. Core intrinsic rotation has generally been observed to behave quite simply in the high confinement mode (H-mode), scaling linearly in the co-current direction with the plasma confinement or, perhaps more accurately, with the pedestal ion temperature 
gradient [1, 2]. In the low confinement mode (L-mode), on the other hand, the core rotation displays significantly more complicated, though reproducible, behavior with dependencies on the electron density, magnetic topology, plasma current, and magnetic field, see for example [3, 4]. As a result, intrinsic rotation experiments have traditionally been classified as either "L-mode" or "H-mode" and the analyses of the data in these two categories kept quite separate.

Recent results from ASDEX Upgrade (AUG), however, have suggested a new interpretation of core tokamak intrinsic rotation that can perhaps merge both L- and H-mode rotation observations into a single consistent story [5]. These results show that in AUG the core intrinsic rotation, regardless of the type of heating method or the confinement regime, is determined by the rotation gradient around mid-radius, which in turn is set by local plasma parameters. In particular, counter-current intrinsic rotation is only observed in the presence of large normalized logarithmic electron density gradients which, due to turbulent particle transport considerations, only occur near the boundary between the ion temperature gradient (ITG) and trapped electron mode (TEM) turbulence regimes [6, 7].

The AUG intrinsic rotation database is set apart from other devices in so far as little effect of the edge can be seen in the core toroidal rotation behavior. Assuming that the generally observed (in the absence of large ripple or applied magnetic perturbations) cocurrent edge rotation is due to an edge localized residual stress torque proportional to the ion temperature gradient [2, 8], the lack of change in the AUG edge data can potentially be explained by the dominant electron heating present in all AUG intrinsic rotation scenarios. The edge ion temperature at $\rho_{\phi} \sim 0.9$ shows little variation within this dataset regardless of L- or H-mode, though detailed pedestal profiles are not available. In the absence of a strong edge localised residual stress torque, core localized intrinsic torques are able to dominate the rotation profile, as is likely the case in L-mode plasmas on other devices. With this picture, it would seem more appropriate to describe tokamak intrinsic rotation behavior as a combination of core and edge localized intrinsic torques coupled, of course, with diffusive and convective momentum fluxes. Within this framework, AUG is an ideal machine in which to study the parameter dependencies of the core-localized intrinsic torques as the behavior is inherently separated from the edge.

Ohmic L-mode discharges present a particularly interesting subset of plasmas as dramatic changes in the energy, particle, and momentum transport have all been reported in this operating regime. In many tokamaks, the Ohmic energy confinement time, $\tau_{E}$, has been observed to scale linearly with increasing density up to a certain point, above which it saturates [9, 10, 11, 12, 13]. These two regimes, above and below the critical density, are known as the linear and saturated Ohmic confinement regimes (LOC/SOC). In addition, on several devices the core rotation [14, 3, 5] and electron density peaking [15, 5] have also been observed to change behavior across the LOC to SOC transition. All of these changes are likely connected to changes in the plasma turbulence, which also transitions from a trapped electron mode (TEM) to an ion temperature gradient (ITG) dominated regime with increasing plasma collisionality 
[16]. The behavior of the electron density profile in this context is particularly well understood [6], and can even be quantitatively reproduced via gyro-kinetic modeling [17, 7].

In this work, the behavior of the intrinsic rotation and the connections between the energy, particle, and momentum transport channels will be explored in ASDEX Upgrade Ohmic L-mode plasmas. For this purpose, the AUG intrinsic rotation database was expanded via dedicated discharges during the 2011-2012 experimental campaign to include a large number of measurements in Ohmic L-mode plasmas. Section [I] describes the diagnostic technique used in AUG to measure the intrinsic toroidal rotation profiles and section III presents the behavior of the rotation throughout the LOC to SOC transition. In Section IV the CXRS measurements of the LOC to SOC toroidal rotation changes are benchmarked against the perpendicular rotation measured by the Doppler reflectometer. Section $\mathrm{V}$ presents the database analysis of all of the Ohmic L-mode intrinsic rotation measurements, the results of which are then compared to a simple model for the prediction of the residual stress in Section VI. Finally, this work is summarized in Section VII

\section{Diagnostic technique}

On AUG intrinsic toroidal rotation profiles are measured via the combination of fast, $\sim 4 \mathrm{~ms}$, charge exchange recombination spectroscopy (CXRS) measurements and short, $12-16 \mathrm{~ms}$, neutral beam injection (NBI) blips [18]. An example of an NBI blip injected into an Ohmic L-mode plasma is shown in Fig. 1. The typical 'rise' and 'fall' times are on the order of $2 \mathrm{~ms}$. This means that the actual "on-time" of a 16ms NBI blip is closer to $12 \mathrm{~ms}$. For dedicated intrinsic rotation experiments the NBI blips are timed such that the first CXRS frame encompasses the entire rise time of the blip plus $2 \mathrm{~ms}$ of "flat-top." This not only optimises the CXRS signal, but also ensures that the rotation evolution during the NBI blip is captured in as much detail as possible, and simplifies the extrapolation of the measured rotation profiles backward to the time point immediately before the NBI blip.

In Ohmic L-mode discharges these blips do not significantly impact the plasma. There is no visible effect on the plasma density, see Fig. 1 b, though there is a slight increase in the electron temperature and a marked change in the plasma stored energy, see Figs. 1 $\mathrm{d}$ and $1 \mathrm{k}$. For the case shown, as the increases in $T_{e}$ and $T_{i}$ are minimal $(\sim 7 \%)$ at low density, the bulk of the $\sim 25 \%$ increase in $\mathrm{W}_{\mathrm{MHD}}$ is assumed to be due to a fast ion contribution. In Ohmic discharges the impact of the NBI blip on the plasma dissipates completely within 100-150ms, see Fig. 11, enabling CXRS measurements to be repeated with a minimum duty cycle of $200 \mathrm{~ms}$. In all of the discharges included in this work the NBI blips were injected at least $300 \mathrm{~ms}$ apart, and all of the shots were examined to be sure that the derived rotation profiles were truly 'intrinsic' and not affected by a build up of co-current momentum from the NBI blips.

The toroidal rotation profiles corresponding to the NBI blip from Fig. 1 are shown 


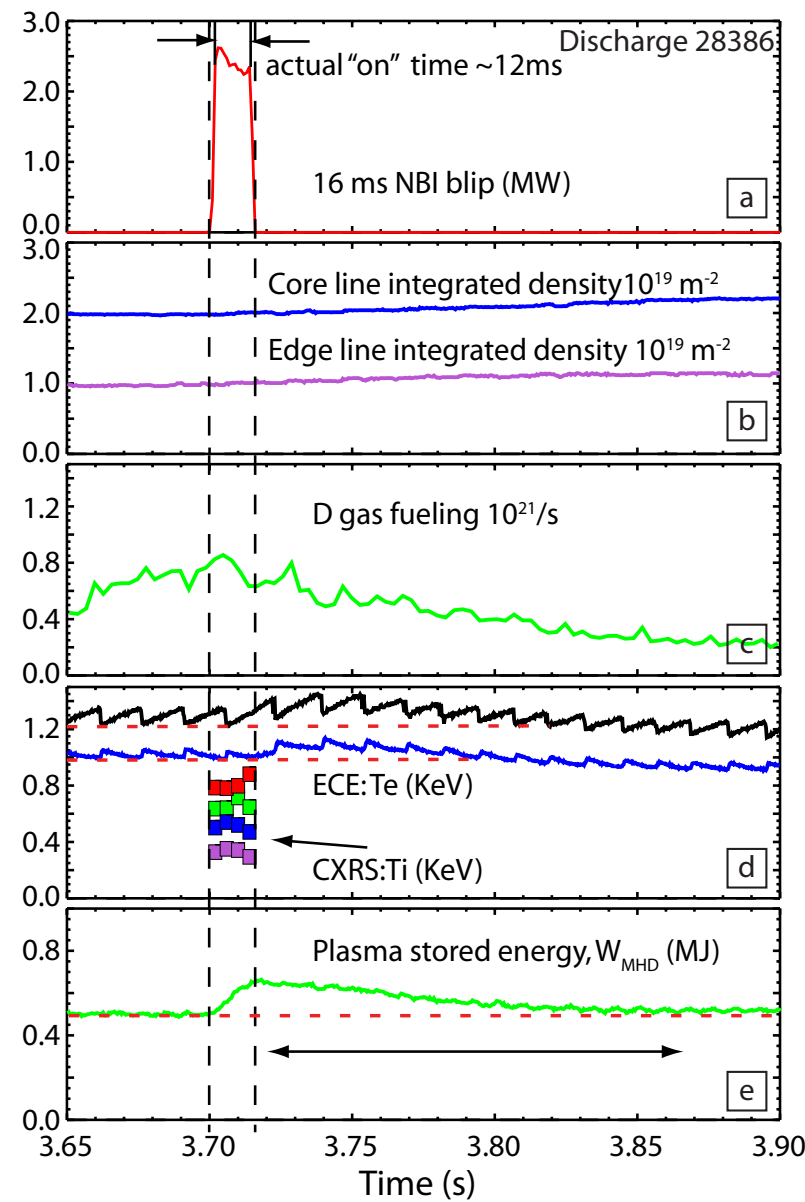

Figure 1. (a) $16 \mathrm{~ms}$ NBI blip injected into Ohmic L-mode discharge 28386 (b) line integrated electron density through the plasma core and edge (c) D gas fueling (d) core and mid-radius $T_{e}$ from the ECE diagnostic and $T_{i}$ from the CXRS diagnostic measured at $\rho_{\phi} \sim 0.7,0.50 .35$, and 0.05 (d) plasma stored energy, $W_{\mathrm{MHD}}$

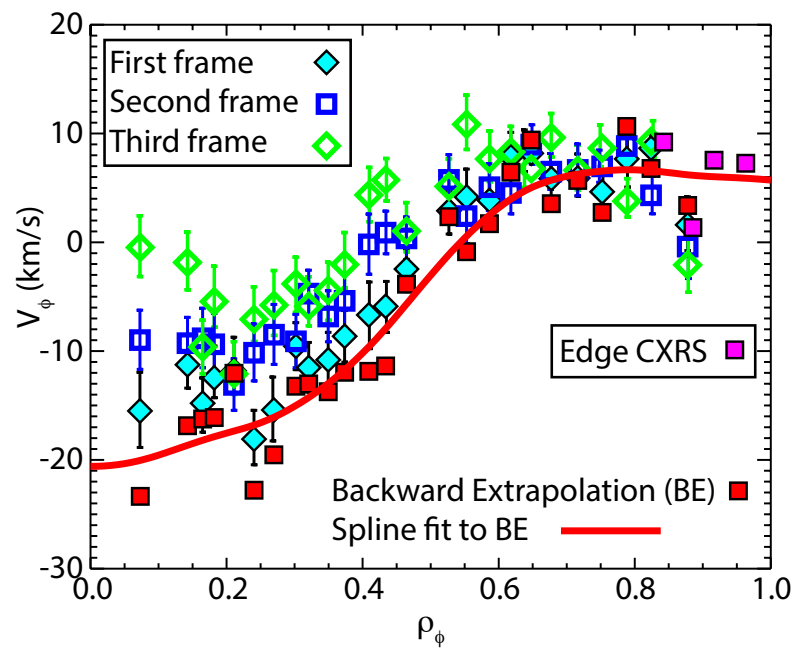

Figure 2. Evolution of the toroidal rotation profile during the 16ms NBI blip shown in Fig. 1. 
in Fig. 2, Here, one can see that even short NBI blips can impart some momentum as the profiles increase in the co-current direction by a few $\mathrm{km} / \mathrm{s}$ throughout the blip. In higher density discharges this rotation increase tends to be reduced compared to the change shown here. Low density Ohmic L-mode plasmas exhibit the maximum observed changes in rotation. Fortunately, in all cases the rotation is observed to change linearly as a function of time enabling the true intrinsic rotation profile to be determined through a simple backward extrapolation technique [19]. Alternatively, a reasonable estimate of the intrinsic rotation can be obtained by averaging the first two to three frames in the NBI blip. The averaging method reduces the scatter in the final rotation profile as compared to the backward extrapolation, resulting in a cleaner set of data. However, it also introduces a small offset of up to $\sim 5 \mathrm{~km} / \mathrm{s}$ to the absolute magnitude in the plasma core $\left(\rho_{\phi}<0.5\right)$.

Previous work on the AUG intrinsic rotation database [19, 5] utilized averaged rather than extrapolated rotation profiles. However, all of the conclusions from these works remain valid and can, in fact, be reproduced by the extrapolated profiles as will be shown in section $\mathrm{V}$. In this work, only backward extrapolated profiles are used. The resultant rotation profiles are then fit with splines and the fits, rather than the data points themselves, are included in the intrinsic rotation database. Note that all of the rotation profiles in this work were measured on the low field side (LFS). In Fig. 2, and in the subsequent sections, the points have been mapped onto the normalized toroidal flux coordinates, $\rho_{\phi}=\sqrt{\left(\psi_{0}-\psi\right) /\left(\psi_{0}-\psi_{\text {sep }}\right)}$, corresponding to their measurement locations, but they do not represent flux surface averaged quantities.

When analysing core rotation profiles one must take care to account for the effect of sawteeth on the rotation profiles as this instability can dramatically alter both the magnitude and the gradient of the profile inside of, and even slightly outside of, the sawtooth inversion radius [20]. For the highest current discharges in the database (1MA) the sawtooth inversion radius is between $\rho_{\phi}=0.28$ and 0.3 . These discharges account for only $10 \%$ of the total points in the database. For the discharges at $0.8 \mathrm{MA}$, which account for a further $30 \%$, the sawtooth inversion radius is observed between $\rho_{\phi}=0.18$ and 0.24 . The bulk of the data points, the remaining $60 \%$, all have lower plasma currents between $0.5 \mathrm{MA}$ and $0.7 \mathrm{MA}$. In many of these discharges the sawtooth inversion radius was difficult to discern, however, the magnetic equilibrium reconstructions indicate a $\mathrm{q}=1$ surface between $\rho_{\phi}=0.15$ and 0.22 . To avoid any possible effect of sawteeth on the interpretation of the database all of the analyses presented in this work focus on radial locations outside of $\rho_{\phi}=0.3$.

It should also be noted that all of the measured profiles correspond to either the boron or carbon impurity population in the plasma, not the main ion species. However, the main ion rotation was calculated via a neoclassical correction [21] to the impurity rotation for a subset of the data points and found to be within $3 \%$ of the impurity rotation. Therefore, in this work no distinction is drawn between the main ion and impurity ion rotation. 


\section{Intrinsic rotation across the LOC-SOC transition}

The 2011 AUG intrinsic rotation database was formed almost entirely from piggy back experiments; very few dedicated discharges were performed. This resulted in a very diverse set of points, which ultimately demonstrated the robustness of the rotation behavior against confinement and heating changes and its reproducibility as a function of local plasma parameters [5]. However, it also resulted in distinct gaps in the explored parameter space and an inability to investigate trends within individual discharges. For this purpose, Ohmic L-mode discharges are of particular interest due to their rich phenomenology and clear connections between the turbulent energy, particle and momentum transport channels.

To study these connections in detail, an AUG Ohmic L-mode intrinsic rotation database, comprised of some 190 profiles, was assembled through dedicated discharges during the 2011-2012 experimental campaign. In these discharges the electron density was ramped to initiate a LOC-SOC transition and the rotation behavior was monitored via NBI blips every $300 \mathrm{~ms}$. This experiment was repeated for a variety of plasma current and magnetic field combinations with the current, $I_{p}$, varying from $0.5 \mathrm{MA}$ to $1 \mathrm{MA}$, and the magnetic field, $B_{T}$, from $-1.5 \mathrm{~T}$ to $-2.5 \mathrm{~T}$. This yielded a range of $q_{95}$ values from -4 to -8.5 . In all cases, the experiments were performed in similarly shaped lower single null plasmas with a mean elongation of $1.62 \pm 0.04$ and an average triangularity $\delta=\left(\delta_{u}+\delta_{l}\right) / 2=0.20 \pm 0.01$. The major radius for these plasmas was on average $1.63 \mathrm{~m}$ $\pm 0.01 \mathrm{~m}$ and the minor radius was $0.52 \mathrm{~m} \pm 0.02 \mathrm{~m}$. Key parameters from one of the discharges in this series of experiments are shown in Fig. 3.

This discharge was performed at $I_{p}=0.5 \mathrm{MA}$ and $B_{T}=-1.5 T\left(q_{95}=4.9\right)$ and the density was increased by means of active feedback on the central line integrated density. This results, unfortunately, in an unsteady increase in the density. However, most attempts to increase the density evenly by means of a slow, steady gas-ramp resulted in fueling to the Ohmic density limit and disruption before the desired measurements could be made. One should note that the periodic increases in the electron density seen in Fig. 3b correlate with the D gas puff shown in 3r, and not with the NBI blips.

The toroidal rotation behavior observed in this discharge (Fig. 3if) is typical of the LOC-SOC transition in AUG and consistent with the perpendicular rotation changes observed by the core Doppler reflectometry, see [22] and section IV] At low densities, and hence high $T_{\mathrm{e}} / T_{\mathrm{i}}$ and low collisionality, the toroidal rotation is co-current across the entire profile. As the density is increased, however, the core rotation reverses direction, while the edge remains constant, creating a hollow rotation profile with a null point around mid-radius. Unfortunately, due to the limited time resolution inherent in the beam blip technique $(\sim 300 \mathrm{~ms})$, it is not possible to determine on what time scale this reversal actually takes place. In the example shown, only one "intermediate" rotation profile at 3.1s was captured. The time period containing the rotation reversal is highlighted in red in Fig. 3.

The actual toroidal intrinsic rotation profiles corresponding to this reversal are 


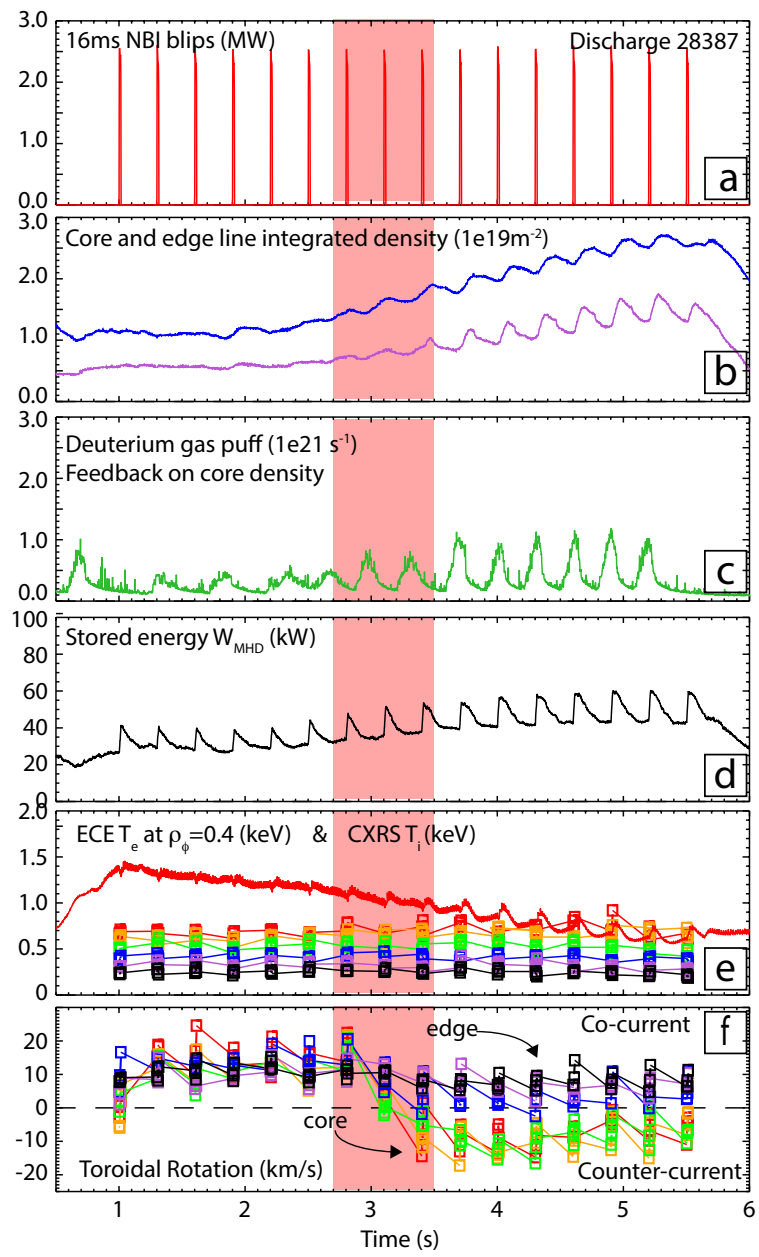

Figure 3. Ohmic L-mode discharge 28387 performed with a density ramp to investigate the rotation change across the LOC-SOC transition. (a) NBI blips (b) core and edge line integrated density (c) deuterium diverter gas puff (d) plasma stored energy $\mathrm{W}_{\mathrm{MHD}}$ (e) electron and ion temperature (f) toroidal rotation. The CXRS measurements shown span the radial range $0.05<\rho_{\phi}<0.8$. The time period of the rotation reversal is indicated by red highlighting.

shown in the top panel of Fig. 4. The data points are the backward extrapolated CXRS measurements and the lines are the spline fits to the data. The error bars represent the error of the spline fit to the data points by taking into account the standard deviation of the fit to the data in fixed radial intervals $(\sim 7.5 \mathrm{~cm})$ centered around each measurement point. Here, one can see that the edge rotation is the same within error bars for all three cases and that the profiles diverge inside of $\rho_{\phi}=0.6$ with the largest changes in gradient occurring in the region $0.3<\rho_{\phi}<0.5$. This is indicative that the greatest residual stress contribution to the momentum flux occurs between these radii and motivates the examination of the Ohmic database there as well.

In Fig. 4 the edge rotation remains quite constant at roughly $5 \mathrm{~km} / \mathrm{s}\left(\rho_{\phi}>0.7\right)$. In fact, within the entire Ohmic database the edge rotation shows very little variation in this region with a mean value of $5 \mathrm{~km} / \mathrm{s} \pm 3 \mathrm{~km} / \mathrm{s}$ and similarly sized error bars on 


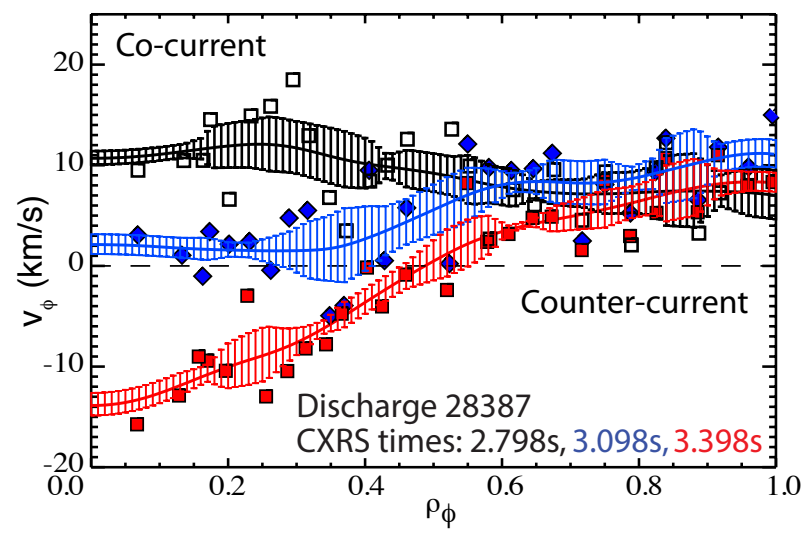

Figure 4. Intrinsic toroidal rotation reversal in Ohmic L-mode discharge 28387. The lowest collisionality profile is shown in black hollow squares, blue diamonds indicate the intermediate profile and the red full squares the highest collisionality profile.

the measured data points. Due to this lack of variation, neither the edge rotation nor the edge Mach number $(0.03 \pm 0.02)$ show any clear trends with any particular parameter or edge gradient outside of the scatter. It is unfortunate that no information on edge intrinsic torque scalings can be extracted from this dataset, however, the constant edge rotation is also advantageous to core intrinsic rotation studies as it translates, practically speaking, into a constant, low, co-current Coriolis pinch offset [23, 24] to the rotation profiles, as will be shown later in section VI. This means that in AUG the core intrinsic rotation behavior is effectively entirely determined by a balance between the diffusive momentum flux and the residual stress $\chi_{\phi} u^{\prime} \sim-\Gamma_{R S}$. Here, $\chi_{\phi}$ is the momentum diffusivity, $u^{\prime}=-\left(R^{2} / v_{t h, i}\right) d \omega / d r$ is the normalised toroidal rotation gradient, $v_{t h, i}=\sqrt{2 T_{i} / m_{i}}$ is the main ion thermal velocity and $\omega$ is the angular velocity given by $V_{\phi}=\omega R$.

Note that when the convective contribution is small, as is the case here, the rotation gradient becomes directly proportional to the turbulence induced residual stress making this the parameter of interest and not the rotation itself. In addition, in Fig. 4 one can see that the sign of the rotation gradient changes before the rotation does. The intermediate profile already has a negative slope around mid-radius with small but cocurrent rotation. This shows that the change in the residual stress takes place at slightly lower collisionality (density) than one would conclude by examining the rotation only.

\section{III.1. Discussion of physics behind intrinsic rotation reversals}

In Ohmic plasmas the toroidal rotation and, more importantly, the rotation gradient are observed to change dramatically with what appears to be relatively minor changes in plasma parameters, i.e. small increases in the plasma density and collisionality. As no changes in the MHD activity are observed concurrent with the rotation reversals (in the discharge shown in Figs. 3 and 4 there is no core MHD activity at all except for small $\sim 0.1 \mathrm{kHz}$ sawteeth) they are presumed to be due to changes in the radial flux of parallel 
momentum generated by the plasma turbulence. Within an electrostatic framework this flux can be written as

$$
\Gamma_{\|}=\oint \int \tilde{v}_{E}^{r} \tilde{f} m v_{\|} d^{3} v d s
$$

where $\tilde{v}_{E}^{r}$ is the radial component of the perturbed $E \times B$ velocity, $\tilde{f}$ is the perturbed velocity distribution, $m$ is the ion mass, $v_{\|}$is the velocity parallel to the magnetic field, and $s$ is the field aligned coordinate, see for example [25]. Hence, this equation represents the radial transport of parallel velocity perturbations via the radial component of the fluctuating $E \times B$ drift. One gets a finite radial flux of momentum only if the integral over the field line is non-zero, which is equivalent to the condition that there be an asymmetry in the perturbed velocity distribution $\tilde{f}\left(v_{\|}, s\right) \neq \tilde{f}\left(-v_{\|},-s\right)$. Due to the toroidal symmetry inherent in tokamak configurations, this translates into a breaking of the symmetry in the $r-\theta$ plane, i.e. a poloidal tilt of the turbulence structures away from radial alignment. Many different mechanisms of breaking the parallel symmetry have been identified including, among others, the Coriolis momentum pinch [23, 24] and residual stress pieces such as perpendicular velocity shear [26, 27, 28, 29], up/down asymmetries [30], intensity gradient [31] and profile shearing [32]. The residual stress contributions are distinguished as those pieces of the momentum flux that are not proportional to either the plasma rotation (convective) or to the rotation gradient (diffusive).

A residual stress mechanism that changes sign from the counter-current to cocurrent direction with increasing collisionality would be qualitatively consistent with the experimental observations. With increasing collisionality tokamak plasmas transition from a trapped electron mode (TEM) dominated turbulence regime, in which the turbulence propagates in the electron diamagnetic direction, to an ion temperature gradient (ITG) dominated regime, in which the propagation direction switches to the ion diamagnetic direction. Any residual stress mechanism that is proportional to the propagation direction of the turbulence, such as intensity gradient [31] or profile shearing [32, also changes sign when the plasma transitions from TEM to ITG. However, in order for such a transition to explain the observed rotation reversals the transition from TEM to ITG should take place in the same parameter range in which the reversals are observed.

Unfortunately, at AUG no experimental measurements of the turbulence propagation direction are available in the core. Therefore, it is necessary to rely on gyrokinetic calculations to gain information on the dominant turbulent modes present in the plasmas. In Fig. 5 the evolution of the toroidal rotation profile is compared to the evolution of the frequency of the dominant turbulent mode $\omega_{r}$ at $\rho_{\phi}=0.35$ and $\rho_{\phi}=0.5$ for the same discharge as presented in Fig. 3. Here, $\omega_{r}$ was calculated via linear gyrokinetic calculations [33, 34] at $k_{y} \rho_{i}=0.3$, which corresponds to the peak of the TEM and ITG growth rates. The vertical lines indicate the time points of the rotation profiles shown in Fig. 4. Here, one can see that in the plasma center ITG turbulence dominates and there is little evolution of the mode frequency throughout the 
discharge, only a slight decrease of $\omega_{r}$ towards zero with increasing collisionality. The dominance of ITG turbulence at this radius is due to the low trapped particle fraction. TEM turbulence can not exist without a sufficient trapped particle population. Further out, at mid-radius, the frequency of the dominant mode clearly evolves throughout the discharge, increasing from strongly TEM toward the ion direction as the plasma density and collisionality are increased. However, the switch to the ion diamagnetic direction does not occur until long after (much higher collisionality) the rotation reversal takes place. In the time interval containing the rotation reversal no changes in the frequency of the dominant turbulent mode are predicted at either location. These results, admittedly without experimental verification, indicate that a change of the dominant turbulence regime is not responsible for the observed rotation reversals. This begs the question: if the dominant turbulence regime is not changing, what is?

\section{III.2. Plasma profile evolution during intrinsic rotation reversals}

The magnitude of residual stress momentum fluxes should be proportional to profile gradients or their second derivatives, as is the case for $\mathrm{E} \times \mathrm{B}$ shear. Experimentally, it is possible to measure the first derivative of most profiles with reasonable uncertainties, however, it is rarely possible to do this for the second derivatives. As such, here the evolution of the toroidal rotation gradient (proxy for the residual stress) is compared to the behaviour of local plasma parameters, focusing on the first derivatives only, in an attempt to identify which gradients are important in setting the rotation gradient in AUG Ohmic L-mode plasmas. In particular, the effective collisionality $\left(\nu_{\text {eff }}\right)$, the normalized logarithmic electron density gradient and the ion and electron temperature gradients $\left(R / L_{\mathrm{ne}}, R / L_{\mathrm{Ti}}\right.$ and $\left.R / L_{\mathrm{Te}}\right)$ are investigated. Here, $L_{X}=-X / \nabla X$ and $\nu_{\text {eff }}=\nu_{\text {ei }} /\left(c_{S} / R\right)$, where $c_{S}$ is the sound speed and $\nu_{\mathrm{ei}}$ is the electron-ion collision frequency. More precisely, this parameter is evaluated in the database as

$$
\nu_{\mathrm{eff}}=0.00279 \times\left(15.94-0.5 \log \frac{n_{e}}{T_{e}^{2}}\right) \times \frac{n_{e}}{T_{e}^{2}} R \sqrt{m_{A}} Z_{\mathrm{eff}},
$$

where density is given in units of $10^{19} \mathrm{~m}^{-3}, \mathrm{~T}_{e}$ in $\mathrm{keV}, \mathrm{R}$ is the major radius in meters, and $\mathrm{m}_{A}$ is the mass of the main ion species in amu, here $\mathrm{m}_{A}=2$. This comparison is shown in Fig. 6 for Ohmic discharge 28386 as a function of increasing effective collisionality at $\rho_{\phi}=0.45$. This radius was chosen because in this discharge it features the largest variation in both Mach and u'. However, similar behavior is observed for all parameters at both larger and smaller radii. This discharge was performed with a plasma current of $0.6 \mathrm{MA}$ and a magnetic field of $-1.9 \mathrm{~T}$ giving a $q_{95}$ value of -5.2 and the collisionality was increased as a function of time by means of a gas ramp similar to that shown in Fig. 3 .

In Fig. 6 the Ohmic confinement time is shown in the top left and clearly demonstrates a transition from linear to saturated confinement around an effective collisionality of 0.3. At the same time, the plasma rotation (Fig. 6b) decreases and crosses from the co-current to the counter-current direction following the decrease in 


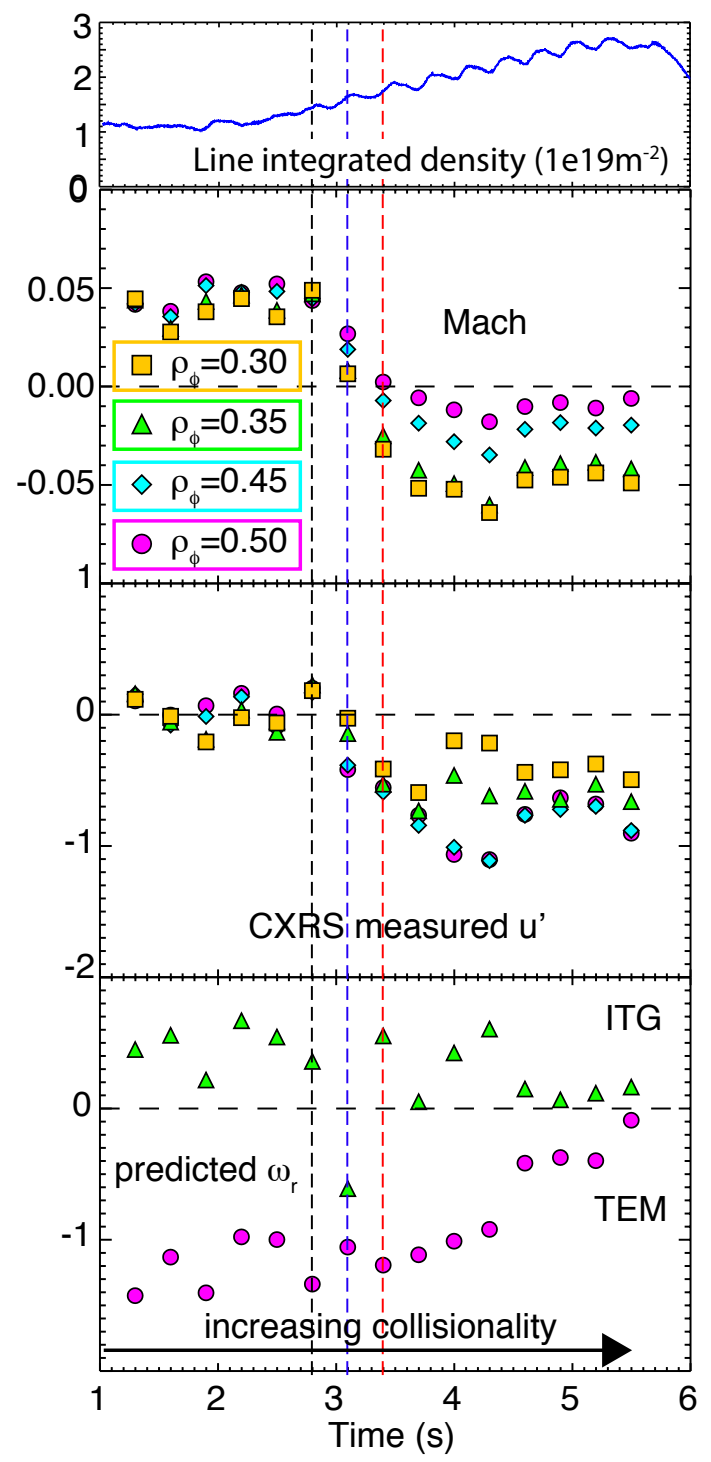

Figure 5. From top to bottom, time evolution of the core electron density, the Mach number, the normalised rotation gradient, and the frequency of the dominant turbulent mode $\omega_{r}$ evaluated at $\mathrm{k}_{y} \rho_{i}=0.3$ as a function of time in discharge 28387. The time points corresponding to the profiles shown in Fig. 4 are indicated by vertical lines.

the rotation gradient u' shown in Fig. 6c. In the SOC regime, as the collisionality is increased even further, the rotation gradient shifts back toward positive values and the Mach number follows suit moving toward the co-current direction. In higher current discharges it is possible to push the collisionality even higher and observe the rotation return to the co-current direction across the entire profile. This full double reversal cycle has been observed in 1MA Ohmic discharges and was already reported on in [5]. Unfortunately, at lower currents it was not possible to get to sufficiently high collisionalities before the Ohmic L-mode density limit was reached and the plasma disrupted. 


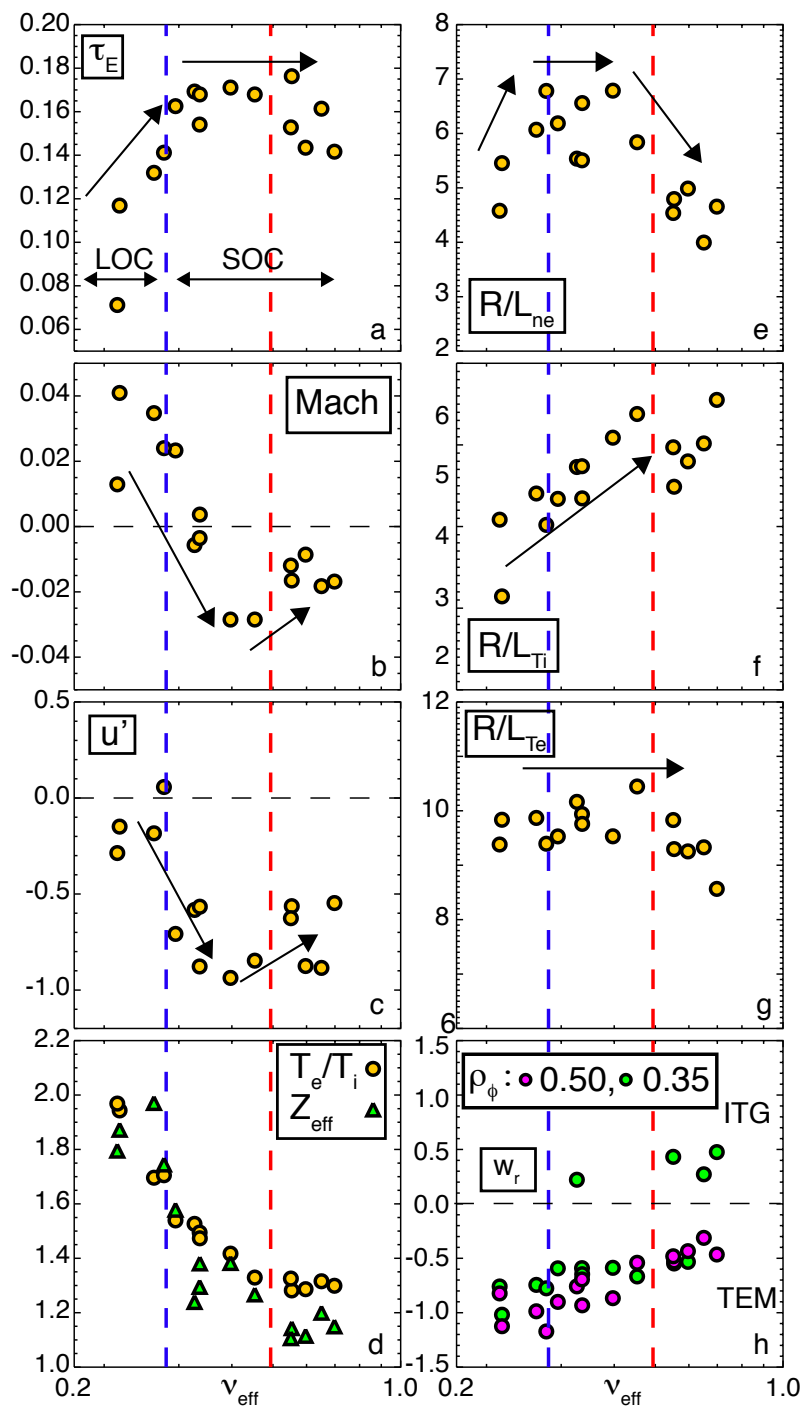

Figure 6. Evolution of the Ohmic confinement time, rotation, and kinetic profiles as a function of increasing effective collisionality at mid-radius in Ohmic L-mode discharge 28386. Left hand side: Global energy confinement time, Mach number, u', $Z_{\text {eff }}$ and $T_{e} / T_{i}$. Right hand side: $R / L_{\mathrm{ne}}, R / L_{\mathrm{Ti}}, R / L_{\mathrm{Te}}$ and $\omega_{r}$. All parameter (except the energy confinement time and $\omega_{r}$ ) were evaluated at $\rho_{\phi}=0.45$. The behavior of the predicted $\omega_{r}$ is given at $\rho_{\phi}=0.35$ and 0.5 .

In panels d-g of Fig. 6 the evolution of $Z_{\text {eff }}, T_{e} / T_{i}$, and the logarithmic electron density, ion temperature, and electron temperature gradients are shown. In addition, the frequency of the dominant turbulent mode as predicted by linear gyrokinetic calculations is shown in the bottom right panel. In the linear Ohmic confinement regime, as the rotation and rotation gradient decrease, $\nu_{\mathrm{eff}}, R / L_{\mathrm{ne}}$ and $R / L_{\mathrm{Ti}}$ all increase. The increase in the ion temperature gradient is consistent with the improvement in the plasma energy confinement and increased equipartition of the ion and electron heat fluxes, and the density peaking is consistent with the idea of the turbulent state moving from the TEM toward the ITG [6]. This will be discussed in more detail in sectionV. Near the transition 
to the saturated confinement regime, the normalized electron density gradient saturates while $\nu_{\text {eff }}$ and $R / L_{\mathrm{Ti}}$ continue to increase concomitant with a continued decrease in the rotation gradient. Finally, deeper in the SOC regime $R / L_{\text {ne }}$ decreases and, at the same time, an increase in the rotation and rotation gradient back toward the co-current direction is observed. Note that $R / L_{\mathrm{Te}}$ stays practically constant during the co- to counter-current rotation reversal demonstrating that this parameter does not contribute to the observed rotation changes. However, the possibility of an $R / L_{\mathrm{Te}}$ dependence of the residual stress in general can not be ruled out by this dataset.

These observations hold for all of the LOC-SOC transitions observed at AUG and lead to the following interpretation: The magnitude of the counter-current directed residual stress momentum flux causing the observed rotation reversals is correlated with $R / L_{\text {ne }}, R / L_{\mathrm{Ti}}$ and collisionality with $R / L_{\text {ne }}$ being the dominant parameter. In the linear Ohmic confinement regime the counter-current directed change in the toroidal rotation is due to the increase in $\nu_{\mathrm{eff}}, R / L_{\mathrm{Ti}}$ and $R / L_{\mathrm{ne}}$. In the saturated Ohmic confinement regime the return of the rotation to the co-current direction is attributed to the decrease of $R / L_{\text {ne }}$, although balanced to some extent by the still increasing collisionality and ion temperature gradient. Thus these parameter dependencies can qualitatively explain the double reversal of the intrinsic rotation observed in AUG Ohmic L-mode density scans. Note that while this data suggests that $R / L_{\text {ne }}$ is the dominant term determining the residual stress in AUG, both $R / L_{\mathrm{Ti}}$ and collisionality also appear to be important parameters particularly when $R / L_{\mathrm{ne}}$ is constant. Hence, these results may still be consistent with those from other devices, which show changes in the rotation profile at constant $R / L_{\mathrm{ne}}$ [35]. The existence and relative strength of these dependencies will be explored further via a database approach in section $\mathrm{V}$.

In Fig. 6 all of the parameters are plotted as a function of the effective collisionality rather than the experimental control parameter, the electron density. This was done because the collisionality was found to be a more fundamental physics parameter in that it organises the experimental data from different plasma currents and magnetic fields better. A similar result was found previously at Alcator C-Mod [36]. In Fig. 7 the rotation reversal data as well as $R / L_{\mathrm{ne}}$ and $R / L_{\mathrm{Ti}}$ from 5 shots with different magnetic field and plasma current configurations are shown together as a function of electron density (left) and $\nu_{\text {eff }}$ (right) at $\rho_{\phi}=0.45$. When these parameters are plotted as a function of the electron density the different shots are clearly separated with the rotation reversals, density peaking, and temperature peaking occurring at different densities for the high and low current shots. However, when the same data is plotted as a function of the effective collisionality, which does not contain any q dependence, the 5 datasets collapse to single curves, albeit with some scatter. These results are in agreement with the previous work from Alcator C-Mod, which showed that the rotation reversal from co-current to counter-current occurs within a very narrow range of collisionality [36]. At Alcator C-Mod the core rotation reversal occurs around $\nu_{\mathrm{eff}}=0.3$ for $0.62 \mathrm{MA}$ discharges and around 0.4 for $1 \mathrm{MA}$ plasmas. This is roughly the same $\nu_{\text {eff }}$ at which the AUG rotation reverses direction (see Figs. 6b and $7 \mathrm{~b}$ ) even though the radial locations 


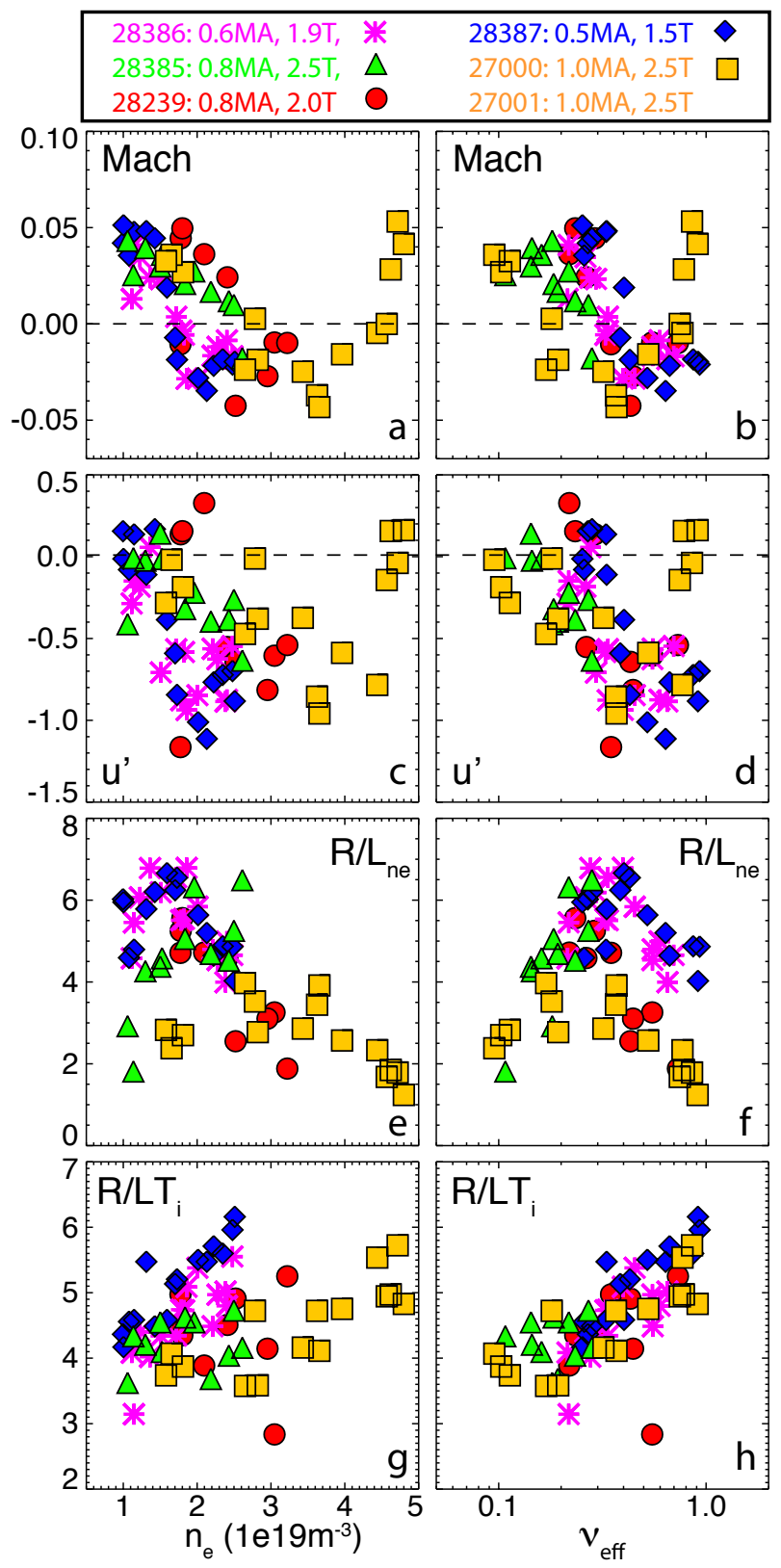

Figure 7. Top to bottom: Mach number, $\mathrm{u}^{\prime}, R / L_{n e}$ and $R / L_{T i}$ as a function of (left) the electron density and (right) effective collisionality at $\rho_{\phi}=0.45$.

plotted and definitions of $\nu_{\text {eff }}$, though similar, are not identical. In the top panels of Fig. 7 the AUG double rotation reversal is clearly visible, particularly for the higher current discharges.

At Alcator C-Mod a core "reversal density" for the transition from co-current to counter-current rotation was found as a function of plasma current and magnetic field as $n_{\mathrm{rev}}=2.8 I_{p} / B^{0.6}$ [4, 36]. It is not possible to apply a scaling of this form to the present set of AUG data due to an insufficient range of magnetic field data (less than a factor of two) and due to the scatter in the data. However, it is worth noting that the 
lower current shots (blue diamonds and purple stars) do show a lower reversal density than the higher $I_{p}$ points consistent with the Alcator C-Mod dataset. The AUG data indicate a reversal density that scales with the local plasma density as $n_{\text {rev }}=3 I_{p}$. The authors do not believe this to be a physically important scaling, rather, it is a useful experimental rule of thumb for obtaining the desired rotation reversals in a given plasma discharge.

\section{Comparison of CXRS and Doppler reflectometry}

It is possible to some extent to cross-check the rotation changes observed by the CXRS diagnostic with the data from the Doppler reflectometer (DR) 37] and potentially even glean information on the phase velocity of the plasma turbulence. The Doppler reflectometer measures the perpendicular rotation $v_{\perp \mathrm{DR}}=v_{\mathrm{ExB}}+v_{\mathrm{ph}}$ in the outer region of the plasma on the low field side using the Doppler shift of the turbulent density fluctuation spectrum at $\mathrm{k}_{\perp}=9 \mathrm{~cm}^{-1}$. If the turbulence phase velocity, $v_{\mathrm{ph}}$, is small compared to the ExB velocity then this system provides a measurement of $v_{\operatorname{ExB}}$, which can be compared to $v_{\mathrm{ExB}}$ calculated from the measured toroidal rotation, ion temperature and density profiles via the radial force balance equation

$$
v_{E x B}=\frac{\nabla p_{i}}{Z_{i} e n_{i} B}+V_{\phi i} \frac{B_{\theta}}{B}-V_{\theta i} \frac{B_{\phi}}{B} .
$$

Here, $B$ is the total magnetic field and $V_{\phi i}, V_{\theta i}, p_{i}, n_{i}$, and $Z_{i} e$ are the toroidal rotation, poloidal rotation, pressure, density and electric charge of a given species, $i$. This comparison is made in Fig. 83. for the same discharge presented in Figs. 3 and 4. Equation 3 was evaluated for the main ion population assuming $n_{i}=n_{e}$ for the diamagnetic term and using the CXRS measured impurity ion temperature profiles. The main ion poloidal rotation was calculated neoclassically using a standard neoclassical formulation [21] and the impurity toroidal rotation was also corrected to represent the main ion species, although this correction was negligible $(\sim 0.2 \mathrm{~km} / \mathrm{s})$. The neoclassical poloidal rotation contribution of $0.5 \mathrm{~km} / \mathrm{s}$ was also found to be relatively unimportant and hence under these assumptions the $\mathrm{E} \times \mathrm{B}$ rotation is determined by the balance between the toroidal rotation and the diamagnetic term. The error bars on $v_{\mathrm{ExB}}$ shown in Fig. 8 are based on the error bars of the spline fit to the CXRS toroidal rotation data (Fig. 4) and a generous $50 \%$ error assumption on the diamagnetic and poloidal velocity terms.

In Fig. 8, $\mathrm{v}_{\perp D R}$ and $v_{\mathrm{ExB}}$ both show qualitatively similar behavior: constant rotation outside $\rho_{\phi} \sim 0.7$ and evolution from the ion diamagnetic to the electron direction with increasing collisionality inside of this radius. There is even good quantitative agreement for the highest collisionality case and for all three cases outside of $\rho_{\phi}=0.7$ and inside of $\rho_{\phi}=0.4$. In the radial region $0.4<\rho_{\phi}<0.7$, however, $\mathrm{v}_{\perp \mathrm{DR}}$ is clearly higher than $v_{\mathrm{ExB}}$ for the two lower collisionality cases. This suggests, at first glance, the presence of a $2 \mathrm{~km} / \mathrm{s}$ turbulent phase velocity in the ion diamagnetic direction, which decreases toward zero as the collisionality is increased. This value is 

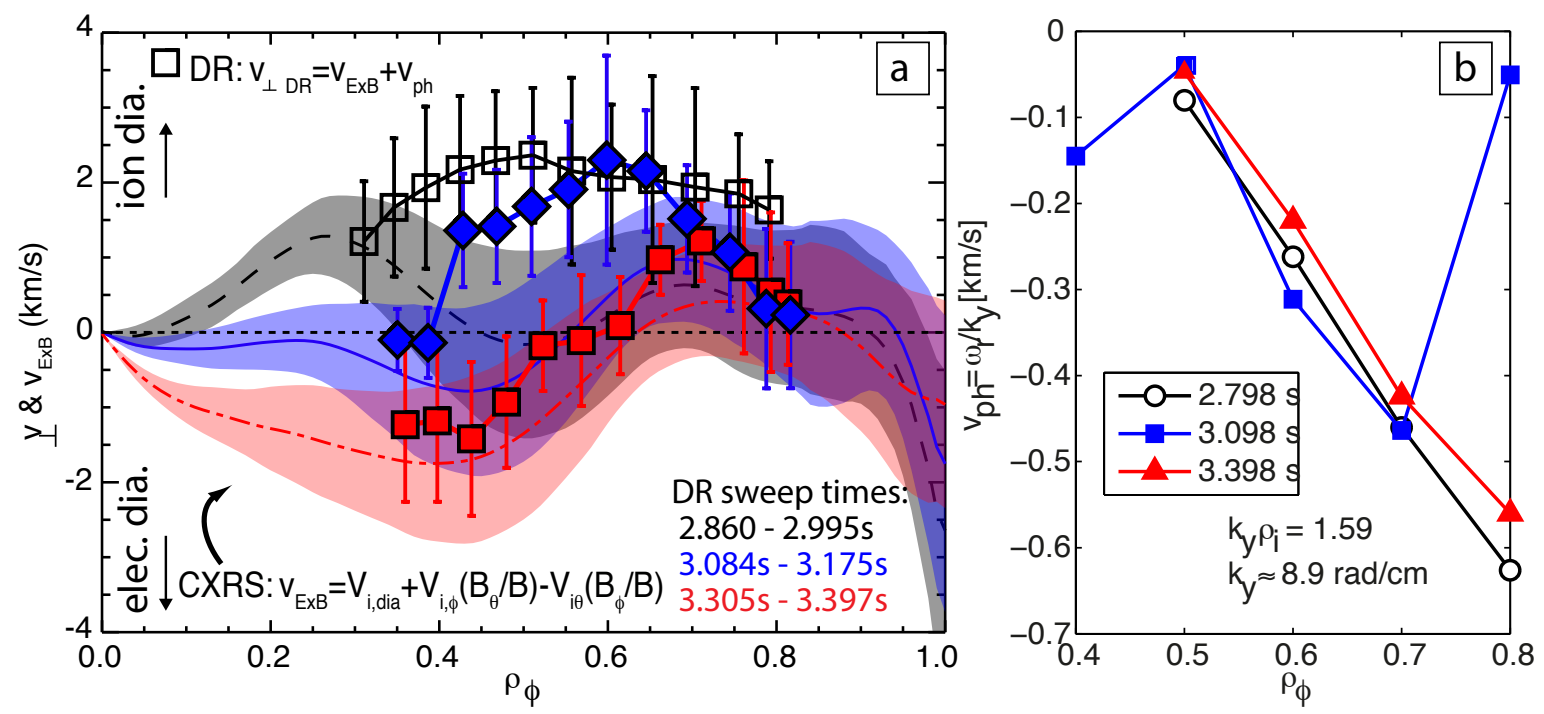

Figure 8. (left) $\mathrm{E} \times \mathrm{B}$ rotation as determined via the main ion radial force balance (lines) and the perpendicular rotation measured by the Doppler reflectometer (symbols). Black hollow squares and dashed line correspond to the lowest collisionality point, blue diamonds with solid blue line are the intermediate case and the red full squares and red dashed-dot line are the highest collisionality point. (right) Phase velocity of the turbulence computed with linear gyrokinetic calculations at $k_{\perp} \rho_{i}=1.59$, which corresponds to the turbulence size-scales measured by the Doppler reflectometer.

significantly larger than expected and the direction is surprising. At these radii TEM turbulence is expected and although ITG and TEM modes are dominant at larger scale lengths than those measured by the DR, one would still expect the phase velocities to be in the same direction. In addition, linear gyrokinetic simulations at the same turbulence size scale as measured by the DR predict turbulence phase velocities between $-0.1 \mathrm{~km} / \mathrm{s}$ and $-0.4 \mathrm{~km} / \mathrm{s}$ (electron diamagnetic direction) for all three cases, see Fig. 8b. This indicates that the perpendicular rotation measured by the DR diagnostic should be a good measurement of the $\mathrm{E} \times \mathrm{B}$ velocity within error bars.

It should be noted that the time windows of the CXRS and the DR measurements (given in Fig. 8), though similar, are not identical. The CXRS measurements correspond to $4 \mathrm{~ms}$ time windows just before the NBI blips, while the DR measurements are made over $90 \mathrm{~ms}$ frequency sweeps. However, none of the plasma profiles vary enough in the corresponding time intervals to account for the level of discrepancy observed between the $\mathrm{DR}$ perpendicular velocity and the calculated $\mathrm{E} \times \mathrm{B}$ velocity. A resolution requires a dramatic change in at least one of the three terms in equation 3: a reduction of the diamagnetic contribution to zero, an increase in the observed toroidal rotation profiles by at least $25 \mathrm{~km} / \mathrm{s}$, an increase in the main ion poloidal rotation in the ion diamagnetic direction by at least a factor of 4 to 5 , up to $2-2.5 \mathrm{~km} / \mathrm{s}$, or a combination of these effects.

The first two possibilities are well outside the experimental uncertainties. Even when the diamagnetic term is varied to the extrema of the density and temperature error bars, this term can not be reduced sufficiently to obtain realistic turbulent phase 
velocities. Completely flat ion temperature and density profiles would be required and are not supported by the data. The uncertainties on the toroidal rotation calibration are better than $\pm 2.5 \mathrm{~km} / \mathrm{s}$ and the measurements are cross-checked against the edge CXRS system, which has rotation uncertainties on the order of $\pm 1 \mathrm{~km} / \mathrm{s}$ [18], and agree well. This leaves only the neoclassical poloidal velocity contribution, which was crosschecked using the NEOART code [38]. For these calculations $Z_{\text {eff }}$ was varied between 1.0 and 2.0 in accordance with the error bars on the IDZ $Z_{\text {eff }}$ measurement for this discharge [39]. Excellent agreement was found between NEOART and the formulation of [21] and the change in $Z_{\text {eff }}$ has very little impact on the resultant main ion poloidal rotation profile as the time points are in the banana regime. Therefore, this analysis suggests that the main ion poloidal rotation at mid-radius is not neoclassical at least for very low collisionalities. This is in disagreement with the conventional wisdom that the poloidal rotation should remain at neoclassical levels due to strong viscous damping, but is qualitatively consistent with recent results from DIII-D which show that at low collisionalities $\left(\nu_{i}^{*}<0.1\right)$ the main ion $(\mathrm{D})$ poloidal rotation is larger than the neoclassical prediction in the diamagnetic direction by up to several $\mathrm{km} / \mathrm{s}$ [40]. However, without direct experimental measurements of the poloidal velocity it is difficult to speculate further. This subject will be the focus of future, dedicated investigations when core poloidal velocity measurements become available.

\section{Analysis of the Ohmic L-mode database}

In order to confirm and quantify the parameter dependencies of the normalised rotation gradient, u', observed in individual discharges (section III.2) a similar analysis to that of [5] was performed on the new Ohmic L-mode intrinsic rotation database. In Fig. 9a-d u' is plotted against the effective collisionality as well as the normalized logarithmic gradients of the electron density, ion temperature, and electron temperature at $\rho_{\phi}=0.35$. The data points in all of the plots are color coded according to their value of $q_{95}$, which is shown in Fig. 9f.

The most readily apparent correlation is between u' and $R / L_{\text {ne }}$, see Fig. 9b. Here, one can see a linear dependence such that positive values of $u$ ' only occur at low values of $R / L_{\mathrm{ne}}$, while the most negative u' points coincide with the highest values of $R / L_{\mathrm{ne}}$. This means that peaked rotation profiles exist together with flat density profiles and that hollow rotation profiles are observed with peaked electron density profiles. Moreover, the central $\left(\rho_{\phi}=0.10\right)$ deuterium Mach number versus u' at $\rho_{\phi}=0.35$, shown in Fig. 9e, demonstrates a clear linear relationship: co-current rotation occurs only for small or positive u' and counter-current rotation with large negative values of u'. Together with 9b this means that co-current rotation only occurs with flat electron density profiles and that the most hollow, counter-current directed core rotation occurs with highly peaked electron density profiles. In Figs. 9a,c, and d possible trends with $\nu_{\text {eff }}$ and the temperature gradients can also be observed. These observations are all in line with the parameter dependencies observed in individual discharges, see section III.2. 

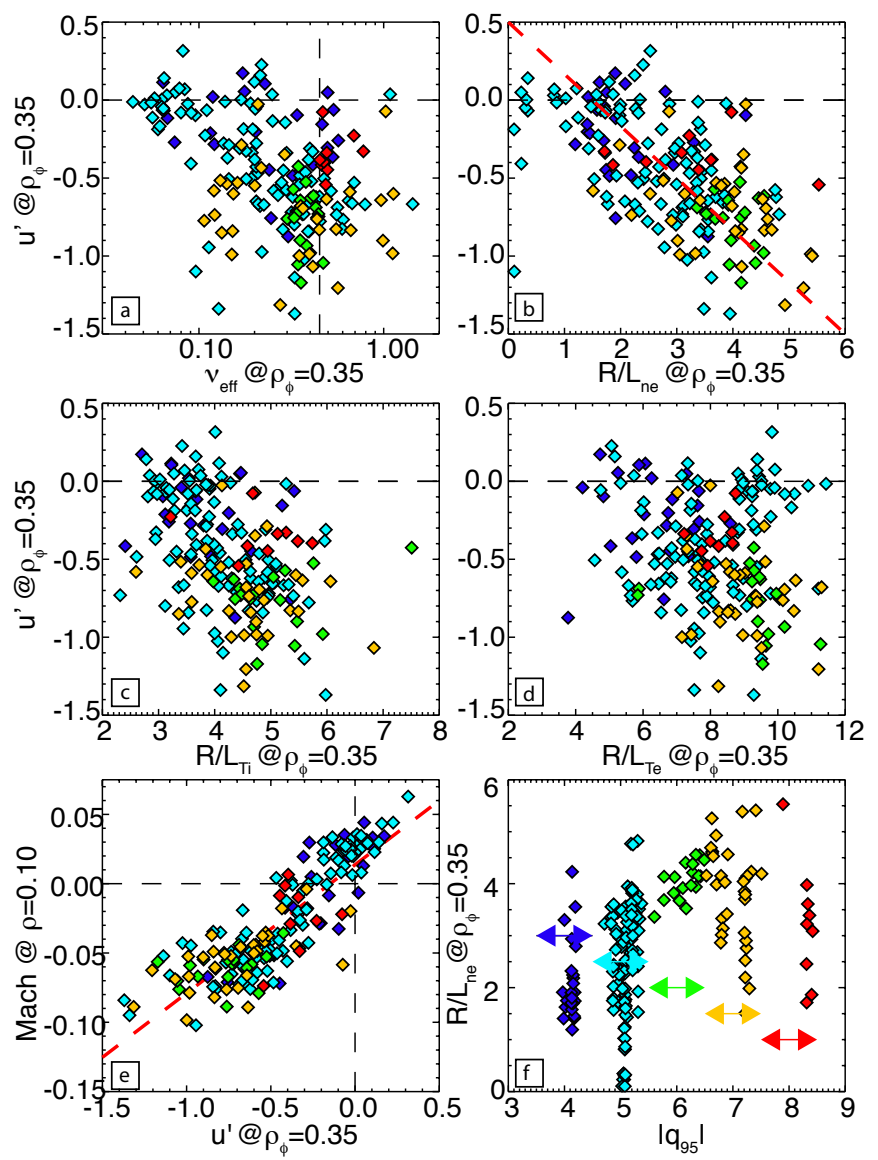

Figure 9. Parameter trends of the AUG Ohmic L-mode intrinsic rotation database evaluated at $\rho_{\phi}=0.35$ (a) u'versus $\nu_{\text {eff }}$, (b) u'versus $R / L_{\mathrm{ne}}$, (c) u'versus $R / L_{\mathrm{Ti}}$ and (d) u' versus $R / L_{\mathrm{Te}}$ (e) D Mach number versus u', and (f) $R / L_{\text {ne }}$ versus q95. The $_{9}$ colors indicate the value of $\mathrm{q}_{95}$, as shown in panel f.

The clear correlation between $\mathrm{u}^{\prime}$ and $R / L_{\text {ne }}$ suggests that the electron density gradient plays a strong role in determining the magnitude of the residual stress momentum flux present in the plasma. Therefore, it is worthwhile to investigate this parameter more thoroughly. In Fig. $10, R / L_{\text {ne }}$ is shown as a function of $\nu_{\text {eff }}$ (top panels) and versus $\omega_{r}$ as calculated via linear gyro-kinetic simulations at $k_{y} \rho_{i}=0.3$ (bottom panels) for both $\rho_{\phi}=0.35$ and $0.5 . R / L_{n e}$ clearly exhibits non-monotonic behavior as a function of both parameters, peaking at a mode frequency of -0.5 (TEM) and at an effective collisionality of roughly 0.4 . This behaviour has been observed before and is well described, even quantitatively reproduced, by gyrokinetic modelling [17, 41, 6]. The circled cloud of points at negative $\omega_{r}$ and high $R / L_{\text {ne }}$ is due to the fact that high $R / L_{\text {ne }}$ itself destabilises TEM modes. Note that the effective collisionality at which the density gradient peaks corresponds roughly to the same value at which the toroidal rotation and rotation gradient are the most counter-current directed, see Fig. 7. Moreover, the similarity in the non-monotonic behavior of these two parameters is highly suggestive of a direct link of the form $u^{\prime} \sim-C R / L_{\text {ne }}$, where $\mathrm{C}$ is a constant. 

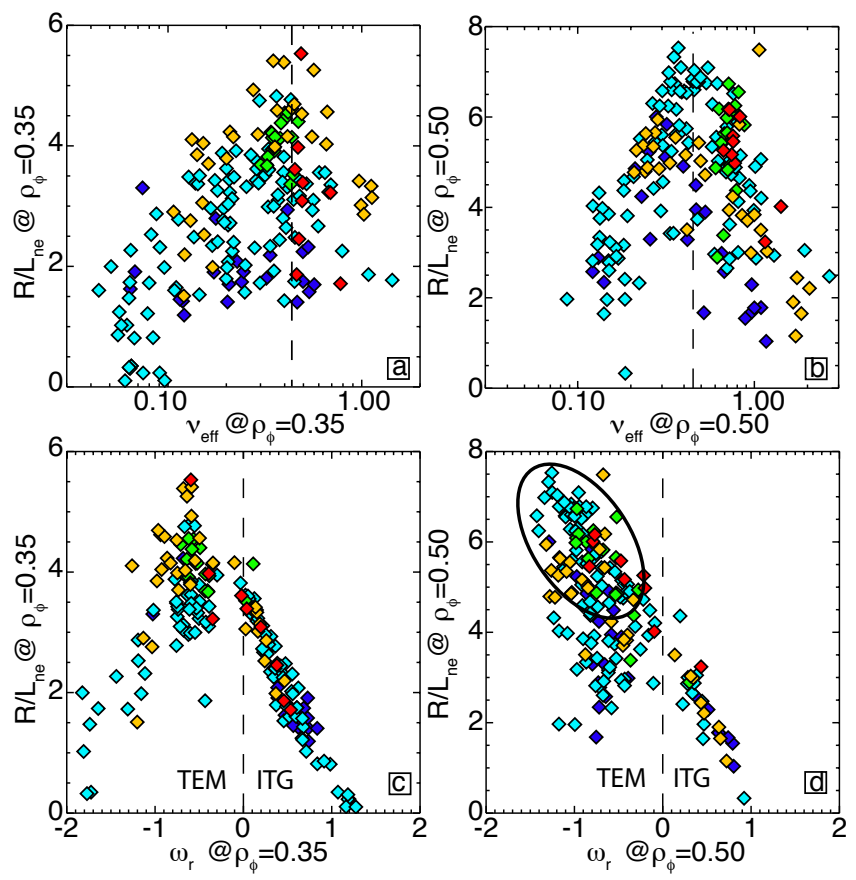

Figure 10. (top) $R / L_{\text {ne }}$ versus $\nu_{\text {eff }}$ and (bottom) $R / L_{\text {ne }}$ versus the calculated real frequency of the dominant turbulent mode at $k_{y} \rho_{i}=0.3$ at $\rho_{\phi}=0.35$ and $\rho_{\phi}=0.5$.

From Fig. 10c one can also see that at $\rho_{\phi}=0.35$ the database is well divided between ITG and TEM data points, while at mid-radius most of the points appear to be TEM dominated (Fig. 10 d). The dominance of TEM is due to the low collisionality, while the more even distribution in the core is a result of the reduced population of trapped electrons. In these plasmas, since the collisionality was scanned by increasing the electron density, which was limited by the Ohmic density limit, it was not possible in most cases to move the plasma solidly into an ITG dominated regime over a broader radial region $\left(0.35<\rho_{\phi}<0.5\right)$. However, $\sim 10 \%$ of the database does meet this criterion and a further $44 \%$ is comprised of discharges that were TEM dominated over this entire radial range. It is interesting to note that in both of these subsets the rotation profiles exhibit gradients covering the full range of the database (co-current and flat to counter-current and extremely hollow). This supports the idea that the plasma profile dependencies are more important in determining the strength and direction of the residual stress torque than which turbulent mode is dominant.

These observations are consistent with the results published in [5] and reproduce the behaviors noted there. The core rotation gradient correlates most strongly with the local electron density gradient scale length. The most hollow counter-current rotation profiles are only observed concurrent with strong electron density peaking, while flatter electron density profiles coincide with co-current rotation. Since the behavior of $R / L_{n e}$ as a function of turbulence regime and collisionality is well understood and is known to peak while the turbulence is still TEM dominated, these results suggest that the co- to counter-current Ohmic L-mode rotation reversals also occur in the TEM regime due to 
profile changes and not a TEM to ITG transition, consistent with the linear gyrokinetic calculations presented in Section III.

The primary differences between this dataset and the 2011 intrinsic dataset are the extension of the density (and $R / L_{\text {ne }}$ ) scans over a wider range of $q_{95}$, the broader range of $I_{p}$ and $B_{t}$ combinations, the extension to higher $R / L_{\mathrm{Ti}}$ values, and of course this dataset is comprised of only Ohmic L-mode points. In the previous dataset, no correlation between $\mathrm{u}^{\prime}$ and either temperature gradient was visible, while in this dataset possible, albeit weaker, correlations are present. In addition, in this dataset a weak linear correlation with $\nu_{\text {eff }}$ is visible, while in the previous dataset a clear nonmonotonic relationship was apparent. This may be due to the absence of both very high collisionality points (low $R / L_{\text {ne }}$ in ITG dominated regime) and extremely low collisionality points (low $R / L_{\text {ne }}$ due to very large $\omega_{r}$ in TEM regime), which in the previous dataset were only achieved through strong ECRH electron heating.

\section{V.1. Multi-variable regression}

To more accurately identify the relative importance of the different parameters a multivariable regression of u' was performed at $\rho_{\phi}=0.35$ and $\rho_{\phi}=0.5$. The results of this regression for $\rho_{\phi}=0.35$, which features the largest range of measured u' values, are given in Eqn. 4 and a comparison of the measured u' values versus the regression is shown in Fig. 11. The regression reproduces the measured data with an RMSE value of 0.27 .

$$
\begin{aligned}
u^{\prime}= & -(0.12 \pm 0.02) \frac{R}{L_{\mathrm{ne}}}-(0.09 \pm 0.04) \ln \left(\nu_{\mathrm{eff}}\right) \\
& -(0.06 \pm 0.03) \frac{R}{L_{\mathrm{Ti}}}-(0.028 \pm 0.014) \frac{R}{L_{\mathrm{Te}}}
\end{aligned}
$$

As expected, the logarithmic electron density gradient is found to be the most statistically significant variable, where the significance is given by the regression coefficient divided by its standard deviation. $R / L_{\mathrm{ne}}$ is followed, in terms of significance, by $\nu_{\text {eff }}$, and then the ion and electron temperature gradients. This is consistent with a visual analysis of Fig. 9 and the behavior observed in individual LOC-SOC rotation reversals. Both $q_{95}$ and $\rho^{*}$ were originally included in the regression. However, the significance of these variables was so low that they were removed. The lack of $\rho^{*}$ dependence is likely due to the lack of variation of this parameter in this dataset. In addition, it is the local $q$ and magnetic shear that are theoretically relevant with respect to the rotation behavior, not $\mathrm{q}_{95}$. At $\rho_{\phi}=0.35$ the local change in the q-profile due to even a large variation in $\left|q_{95}\right|$ (4-8) is likely small. Therefore, it is not surprising that $q_{95}$ does not factor as an important variable. Unfortunately, no measurements of the q-profiles are available in any of these discharges and the equilibrium reconstruction, without MHD constraints, is not sufficiently reliable in the plasma centre to use the local 


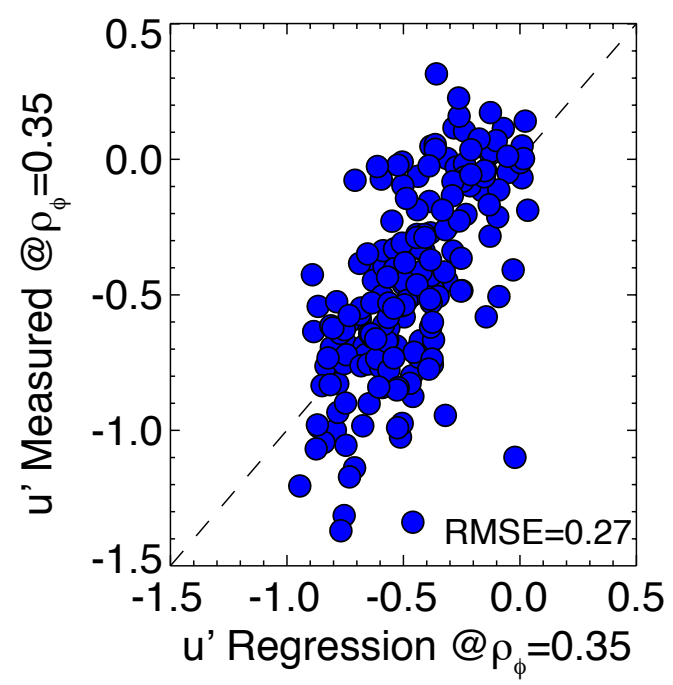

Figure 11. Measured values of u' at $\rho_{\phi}=0.35$ versus the multi-variable regression of u' at the same location, see Eqn. 4.

values in the regression and obtain meaningful results. Variations on this regression using the electron and ion pressure gradients with $p_{\mathrm{e}}=n_{\mathrm{e}} T_{\mathrm{e}}$ and $p_{\mathrm{i}}=n_{\mathrm{e}} T_{\mathrm{i}}$, rather than the temperature and density gradients independently, were also performed. However, no improvement in the quality of the fit was observed.

The statistical relevance rather than the statistical significance, is potentially of more interest to this study. This parameter is given by the regression coefficient times the standard deviation of the corresponding input data (regression variable) and represents the impact of the regression variable on the regressed variable (in this case $\mathrm{u}^{\prime}$ ) independent of all of the other input parameters. The statistical relevance gives the same order yielding the values -0.15 , and -0.07 for $R / L_{\text {ne }}$ and $\nu_{\text {eff }}$, a value of -0.06 for $R / L_{\mathrm{Ti}}$, and -0.04 for $R / L_{\mathrm{Te}}$. The standard deviation of $\mathrm{u}$ ' at this location is 0.35 , meaning the changes in $R / L_{\text {ne }}$ can account for $42 \%$ of the observed changes in u' with $\nu_{\text {eff }}$ accounting for a further $20 \%$. This demonstrates that while changes in the electron density gradient have the largest impact on the rotation gradient, the changes in the other parameters can not be safely ignored.

\section{Comparison to predictions from linear gyrokinetic calculations}

The results from the multi-variable regression of the database can also be compared to predictions of $u^{\prime}$ from local, linear, gyrokinetic calculations [33, 42] by imposing a finite, constant, poloidal tilt angle, $\theta_{0}$, on the turbulence mode structure. Within a linear picture, the most unstable mode is ballooned on the LFS mid-plane and is radially aligned, i.e. $\theta_{0}=0$. However, due to the presence of radial variation in the plasma profiles and equilibrium these modes acquire a poloidal tilt, thus breaking the parallel symmetry of the mode. With respect to momentum transport, including a finite $\theta_{0}$ in the linear calculations affects the obtained growth rates, Prandtl numbers, 
Coriolis pinch numbers and, most importantly, yields a finite residual stress due to the resultant asymmetric eigenfunction, see for example [32]. The degree and direction of the asymmetry is proportional to the poloidal tilt angle used in the calculations. Therefore, the magnitude and direction of the resultant residual stress momentum flux is also linearly determined by the size and sign of $\theta_{0}$.

Simple linear models can not determine the correct $\theta_{0}$; such calculations require much more expensive simulations with more complete numerical tools. However, if a reasonable value of $\theta_{0}$ can be determined by other means (ideally an actual measurement) and imposed in the calculations, then linear codes can deliver the relative strengths of the various momentum flux components, i.e. the ratios $\Gamma_{\mathrm{RS}} \theta_{0} / \chi_{\phi}$ and $R V_{c} V_{\phi} / \chi_{\phi}$. Here, $V_{c}$ is the convective velocity and $\Gamma_{R S}$ is the residual stress piece of the radial flux of toroidal momentum (see the discussion in section III.1) generated by the electrostatic turbulence by imposing a fixed, constant poloidal tilt angles on the turbulent eddies in the linear simulations. In this work, an initial tilt angle $\theta_{i}$ was used for the calculations. However, since the residual stress momentum flux is linear in the tilt angle, this term can be rescaled for any tilt angle by multiplying by $\theta_{0} / \theta_{i}$. Via this approach, predicted normalised rotation gradients can be determined according to

$$
u_{p}^{\prime}=\frac{-R V_{c} V_{\phi}}{\chi_{\phi}}-\frac{\Gamma_{R S}}{\chi_{\phi}} \frac{\theta_{0}}{\theta_{i}} .
$$

These values can then be compared to the actual measured u' data and the averaged $\theta_{0}$ value found that best reproduces the experimental data. The parameter dependencies of the normalised residual stress term can then be examined and compared to the experimentally observed parameter dependencies of $u$ '. Hence, this approach attempts to separate the problem of the prediction of the residual stress in two steps: The identification of the average tilting angle developed by the turbulence and the determination of the parametric dependences displayed by the predicted residual stress using this angle.

Our analysis is intended to test whether the parametric dependences observed in the experiment are at least partly captured by the quasi-linear predictions in the presence of a fixed tilting angle. In addition, since different residual stress mechanisms are expected to produce eddy tilting in opposite directions, this procedure enables systematic differences between observations in different turbulence regimes to be identified. It can be considered complementary to the more appropriate and fundamental approach of applying a non-local, non-linear model. However, it is not possible at present to run such a code over the entire database in a manageable amount of time. Instead we apply a very simple linearized local model, but over a very large set of observations.

As a first step the database is divided into subsets based on location and dominant turbulence regime as determined by the gyrokinetic calculations, see Fig. 10. The optimum tilt angle per dataset is then determined by minimising the root-mean-square difference between the 'predicted' (Eqn. 5) and measured values of u'. This procedure results in an optimum tilt angle of -0.25 radians for the TEM data points at both 

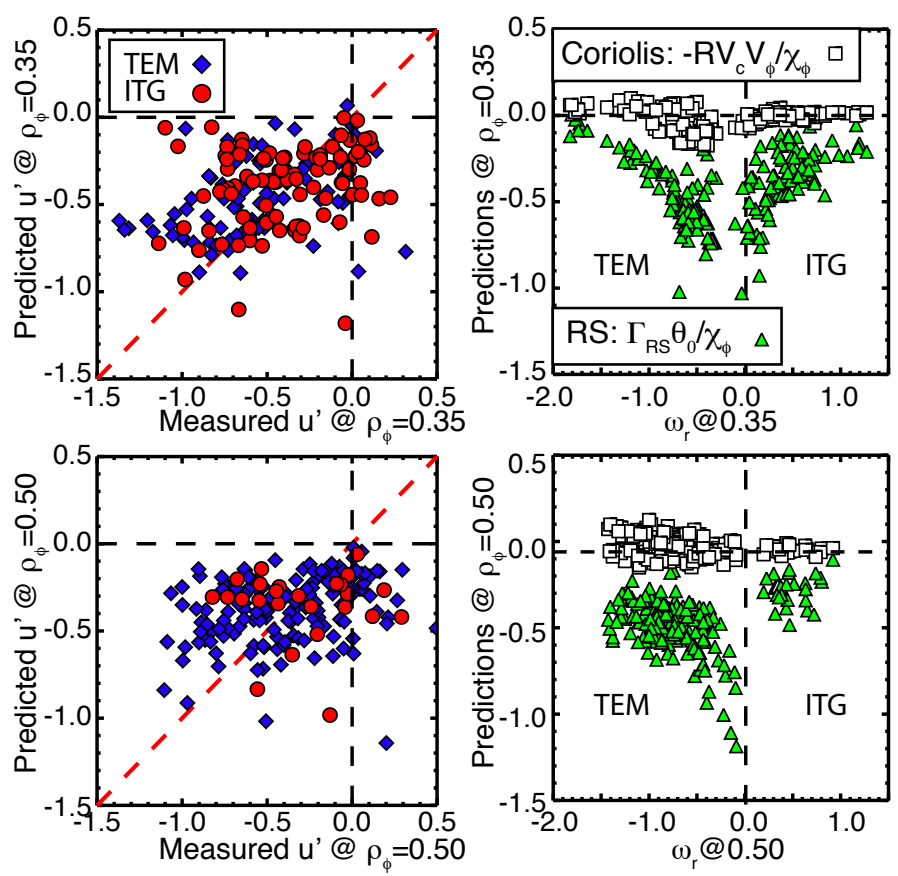

Figure 12. (left)Predicted versus measured values of the normalised rotation gradient at $\rho_{\phi}=0.35$ (top) and 0.5 (bottom) sorted by dominant turbulence regime. ITG points are shown as red circles while TEM points are shown as blue diamonds. (right) Predicted residual stress (green triangles) and Coriolis pinch (white squares) momentum fluxes normalised to $\chi_{\phi}$ at $\rho_{\phi}=0.35$ (top) and 0.5 (bottom). $\theta_{0}=-0.25$ radians was used for the TEM datasets at both radial locations. For the ITG sets $\theta_{0}=-0.175$ and -0.05 radians were used at $\rho_{\phi}=0.35$ and $\rho_{\phi}=0.5$, respectively.

$\rho_{\phi}=0.35$ and 0.5 . The ITG points, on the other hand, require smaller tilt angles of -0.175 radians and -0.05 radians at the same respective locations. Note, the TEM and ITG tilt angles found here are very similar to the ones used in a previous study [5]. The fact that the ITG data points at both radial locations require smaller tilt angles to optimise the quantitative agreement with the measurements suggests that there are at least two main processes contributing to the poloidal tilt of the turbulent eddies: one proportional to the sign of the turbulence propagation, such as profile shearing [32] or intensity gradient [31], and the second independent of $\omega_{r}$, such as $E \times B$ shear [26, 27, 28, 29].

Using these angles the comparison between the 'predicted' and measured u' values is shown on the left hand side of Fig. 12. Here one can see that the averaged $\theta_{0}$ values produce $u_{p}^{\prime}$ values of the correct order of magnitude, but do not perfectly reproduce the measured u' values. An array of 'perfect' $\theta_{0}$ values could of course be computed, but the goal is to test whether the calculated residual stress term with a constant tilt angle (no direct information taken from the measured gradients) can reproduce the observed experimental dependencies. Hence the comparison of the 'predicted' to measured u' values acts only to obtain a residual stress term of the correct size, a scaling factor, but does not influence the parameter dependencies of this term. On the right hand side of 


\begin{tabular}{cccccc}
\hline Regressed Variable & $R / L_{\mathrm{ne}}$ & $\ln \left(\nu_{\mathrm{eff}}\right)$ & $R / L_{\mathrm{Ti}}$ & $R / L_{\mathrm{Te}}$ & $\mathrm{RMSE}$ \\
Measured u' $@ \rho_{\phi}=0.35$ & $-0.12 \pm 0.02$ & $-0.09 \pm 0.04$ & $-0.06 \pm 0.03$ & $-0.03 \pm 0.01$ & 0.27 \\
Predicted u' $@ \rho_{\phi}=0.35$ & $-0.11 \pm 0.01$ & $-0.06 \pm 0.02$ & $-0.01 \pm 0.02$ & $0.01 \pm 0.01$ & 0.16 \\
\hline Measured u' @ $\rho_{\phi}=0.50$ & $-0.05 \pm 0.01$ & $-0.14 \pm 0.03$ & $-0.08 \pm 0.03$ & $-0.03 \pm 0.02$ & 0.28 \\
Predicted u' $@ \rho_{\phi}=0.50$ & $-0.04 \pm 0.01$ & $-0.08 \pm 0.02$ & $-0.06 \pm 0.01$ & $0.01 \pm 0.01$ & 0.15 \\
\hline
\end{tabular}

Table 1. Multi-variable regression coefficients for measured and predicted normalised rotation gradients at $\rho_{\phi}=0.35$ and 0.5 . A fixed, imposed tilt angle of -0.25 radians was used for the predictions of the TEM points at both radial locations. Tilt angles of -0.175 radians and -0.05 radians were used for the ITG points at $\rho_{\phi}=0.35$ and $\rho_{\phi}=0.50$, respectively.

Fig. 12 the calculated residual stress and Coriolis pinch terms using the optimised tilt angles at both radial locations are also shown. Here, one can see that for reasonable $\theta_{0}$ values the residual stress is much larger than the Coriolis pinch, which is on average zero. Hence, $\mathrm{u}^{\prime}$ is effectively given by the residual stress term as was assumed in previous sections $\left(u^{\prime} \sim \Gamma_{R S} / \chi_{\phi}\right)$.

The next step is to compare the parameter dependencies of the predicted residual stress with those determined for the experimental values of $u$ '. Even though the Coriolis pinch term is found to be small $(\sim 0)$, for consistency we still make the comparison between the measured and predicted values of $u^{\prime}$ rather than $\Gamma_{\mathrm{RS}} \theta_{0} / \chi_{\phi}$. The two quantities exhibit nearly identical dependencies. A multi-variable regression as a function of the same experimental parameters $\left(R / L_{\mathrm{ne}}, \ln \left(\nu_{\mathrm{eff}}\right), R / L_{\mathrm{Ti}}\right.$, and $\left.R / L_{\mathrm{Te}}\right)$ was performed on the predicted u'values at two radial locations, $\rho_{\phi}=0.35$ and 0.5 . The results of these regressions, in addition to the experimental coefficients, are given in Table 1. The regressions on the predicted u' values are able to organise the data very well as evidenced by the small RMSE listed in Table 1 .

In the plasma core both the measured data and the predicted values show almost identical dependencies on the logarithmic electron density gradient followed by $\ln \left(\nu_{\text {eff }}\right)$. The weaker dependencies of the measured data on the temperature gradients are not captured by the predictions. In the measured data at mid-radius $R / L_{\text {ne }}$ and collisionality have the highest statistical significances $(\sim 5)$ and the highest statistical relevances, 0.08 for $R / L_{\text {ne }}$ and 0.06 for $\nu_{\text {eff. }}$ Thus, despite the apparent ordering given by the size of the coefficients, the measured data show the strongest sensitivities to $R / L_{\mathrm{ne}}$ and collisionality. Together they can account for almost $50 \%$ of the observed rotation changes at mid-radius. The predicted data on the other hand, despite the misleading similarity in the size of the coefficients, gives the largest significance to $R / L_{\mathrm{Ti}}$ and slightly lower weight to both $R / L_{\text {ne }}$ and $\nu_{\text {eff }}$. The normalised density gradient, however, still retains the largest statistical relevance followed closely by $R / L_{T i}$ accounting for $34 \%$ and $29 \%$ of the predicted u' changes, respectively. Thus, this simple model, despite its obvious limitations, is able to capture the dominant parameter trends observed in the data reasonably well, in particular in the plasma core. 


\section{Summary and Conclusions}

Recent upgrades to the core CXRS diagnostic have significantly improved the quality and time resolution of the AUG toroidal rotation and ion temperature measurements. This has enabled AUG to contribute in a meaningful way to on-going intrinsic rotation and momentum transport studies. In the 2011-2012 experimental campaign the AUG intrinsic rotation database was expanded to include a large number of measurements from Ohmic L-mode discharges covering a wide range of plasma densities, currents and magnetic fields. In particular, the rotation behavior across the transition from the linear to saturated Ohmic confinement regimes was studied and compared to the plasma profile evolution and to linear gyrokinetic simulations of the plasma turbulence.

In AUG, at low density, and hence low collisionality, the core intrinsic rotation is in the co-current direction. This, in conjunction with the co-current edge rotation seen routinely in LSN AUG discharges, gives a flat or slightly peaked co-current rotation profile. As the plasma density is increased, however, the core rotation decreases eventually resulting in a hollow, counter-current profile with a null point around midradius. When the density is increased even further, a second reversal back toward the co-current direction occurs. In all cases, the edge rotation, into a radius of about $\rho_{\phi}=0.7$, remains small, co-current and constant within error bars. This implies that the Coriolis pinch contribution to the momentum flux is also small (supported by linear gyrokinetic calculations, see Section $\mathrm{VI}$ ) and that the core intrinsic rotation behavior is determined by a balance between the diffusive momentum flux and the core localised residual stress $u^{\prime} \sim-\Gamma_{R S} / \chi_{\phi}$. This makes the rotation gradient a better proxy for the residual stress than the rotation itself.

The observed toroidal rotation reversal from co- to counter-current could be explained by a change of the dominant turbulence regime from TEM to ITG as several residual stress mechanisms that are proportional to the sign of the turbulence propagation direction have been identified. However, linear gyrokinetic calculations of the dominant turbulent mode frequency do not indicate a change in the dominant turbulence regime in the same parameter regime in which the rotation reversals occur. Furthermore, the second reversal of the rotation back to the co-current direction at even higher collisionality could not be explained by such a mechanism. Therefore, the evolution of the toroidal rotation gradient was compared to additional theoretically relevant plasma parameters including the effective collisionality, $R / L_{\mathrm{ne}}, R / L_{\mathrm{Ti}}$ and $R / L_{\mathrm{Te}}$. This analysis revealed that increasing counter-current rotation is correlated with increasing $\nu_{\mathrm{eff}}, R / L_{\mathrm{Ti}}$, and $R / L_{\mathrm{ne}}$ with $R / L_{\mathrm{ne}}$ being the dominant parameter. Indeed the observed dependence on $R / L_{\text {ne }}$ can qualitatively explain the second rotation reversal back to the co-current direction as the electron density is observed to first peak with increasing collisionality and then decrease again consistent with the changes observed in the rotation profile. It is clear, however, that even though $R / L_{\text {ne }}$ appears to be the dominant parameter, other terms can and do affect the rotation profile, particularly when $R / L_{\text {ne }}$ is constant. Therefore, the AUG results may still be consistent with results 
from other experiments which show changes in plasma rotation with increasing electron density $\left(\nu_{\text {eff }}\right)$ at constant $R / L_{\text {ne }}[35$.

In order to confirm these results and to provide more quantitative information a multi-variable regression was performed on the new Ohmic L-mode database as a function of the same parameters. The results of this analysis were consistent revealing that in the plasma core the normalized rotation gradient depends most strongly on $R / L_{\text {ne }}$ followed by the effective collisionality and then the temperature gradients. This lead to the examination of the normalised electron density gradients which show a clear non-monotonic dependence as a function of both $\nu_{\text {eff }}$ and $\omega_{r}$ with the peak on the TEM side, consistent with predictions from turbulent particle transport theory [6]. These results suggest that the co- to counter-current Ohmic L-mode rotation reversal also occur in the TEM regime due to profile changes and not at the TEM-ITG transition. Within this picture the second rotation reversal back to co-current values is explained by the subsequent flattening of the electron density profile as the turbulence regime transitions from TEM to ITG.

Lastly, a simple model for the residual stress utilising linear gyrokinetic calculations with an imposed, constant, poloidal tilt angle of the turbulent eddies was applied to the database. This simple model, despite its obvious limitations, is able to capture the dominant parameter trends observed in the data reasonably well. Moreover, it demonstrated that ITG data points are best described with a reduced poloidal tilt angles compared to the TEM points. This indicates that there are at least two main processes contributing to the poloidal tilt of the turbulent eddies: one proportional to the sign of the turbulence propagation, such as profile shearing [32] or intensity gradient [31], and the second independent of $\omega_{r}$, such as $E \times B$ shear [26, 27, 28, 29].

\section{Acknowledgments}

The authors would like to acknowledge the efforts and contributions of the entire ASDEX Upgrade team and in particular the NBI and ECH groups. This work was in part supported by the European Communities under the contract of Association between EURATOM/IPP and carried out within the framework of the EFDA Workprogramme 2012 Transport Task Agreement WP12-TRA-04. The views and opinions expressed herein do not necessarily reflect those of the European Commission. 
[1] J. E. Rice et al. Nucl. Fusion, 47:1618, 2007.

[2] J. E. Rice et al. Phys. Rev. Lett., 106:215001, 2011.

[3] B. P. Duval et al. Phys. Plasmas, 15:056113, 2008.

[4] J. E. Rice et al. Nucl. Fusion, 51:083005, 2011.

[5] C. Angioni et al. Phys. Rev. Lett., 107:215003, 2011.

[6] E. Fable et al. Plasma Phys. Control. Fusion, 52:015007, 2010.

[7] C. Angioni et al. Nucl. Fusion, 52:114003, 2012.

[8] K. Ida et al. Nucl. Fusion, 50:064007, 2010.

[9] R. R. Parker et al. Nucl. Fusion, 25:1127, 1985.

[10] R.V. Bravenenc et al. Plasma Phys. Control. Fusion, 27:1335, 1985.

[11] F. X. Soldner et al. Phys. Rev. Lett, 61:1105, 1988.

[12] F. Wagner and U. Stroth. Plasma Phys. Control. Fusion, 35:1321, 1993.

[13] G. Bracco and K. Thomsen . Nucl. Fusion, 37:759, 1997.

[14] J. E. Rice et al. Nucl. Fusion, 53:033004, 2013.

[15] C. Angioni et al. Phys. Plasmas, 12:040701, 2005.

[16] F. Romanelli et al. Nucl. Fusion, 26:1515, 1986.

[17] C. Angioni et al. Nucl. Fusion, 51:023006, 2011.

[18] E. Viezzer et al. Rev. Sci. Instrum., 83:103501, 2012.

[19] R. M. McDermott et al. Plasma Phys. Control. Fusion, 53:124013, 2011.

[20] B. P. Duval et al. Proc. 23rd IAEA Fusion Energy Conf.(Daejeon, Korea, 1116 October) EXS/P4-01 http://infoscience.epfl.ch/record/153794/files/107803008.pdf, 2010.

[21] Y. B. Kim et al. Phys. Fluids B., 3:2050, 1991.

[22] G. D. Conway et al. Nucl. Fusion, 46:S799-S808, 2006.

[23] A. G. Peeters, C. Angioni and D. Strintzi. Phys. Rev. Lett., 98:265003, 2007.

[24] T. S. Hahm et al. Phys. of Plasmas, 14:072302, 2007.

[25] A. G. Peeters et al. Nucl. Fusion, 51:094027, 2011.

[26] R. R. Dominguez and G. M. Staebler. Phys. Fluids B., 5:3876, 1993.

[27] X. Garbet et al. Phys. Plasmas, 9:3893, 2002.

[28] O. D. Gurcan et al. Phys. Plasmas, 14:042306, 2007.

[29] F. Casson et al. Phys. Plasmas, 16:092303, 2009.

[30] Y. Camenen et al. Phys. Rev. Lett., 102:125001, 2009.

[31] P. H. Diamond et al. Nucl. Fusion, 49:045002, 2009.

[32] Y. Camenen et al. Nucl. Fusion, 51:073039, 2011.

[33] W. Dorland et al. Phys. Rev. Lett, 85:5579, 2000.

[34] M. Kotschenreuther et al. Comput. Phys. Commun., 88:128, 1995.

[35] M. L. Reinke et al. Plasma Phys.Control. Fusion, 55:1012001, 2013.

[36] J. E. Rice et al. Phys. Plasmas, 19:056106, 2012.

[37] G. D. Conway et al. Plasma Fusion Res., 5:S2005, 2010.

[38] A. G. Peeters et al. Phys. Plasmas, 7(1):268, 2000.

[39] S. Rathgeber et al. Plasma Phys. Control. Fusion, 52:095008, 2010.

[40] B. A. Grierson et al. Nucl. Fusion, 53:063010, 2013.

[41] C. Angioni et al. Plasma Phys. Control. Fusion, 51:124017, 2009.

[42] N. Kluy et al. Phys. Plasmas, 16:122302, 2009. 